\title{
Dyadic Interference Leads to Area of Uncertainty During Face-to-Face Cooperative Interception Task
}

\author{
Charles Faure*, Annabelle Limballe, Anthony Sorel, Théo Perrin, Benoit Bideau and \\ Richard Kulpa*
}

Univ Rennes, Inria, M2S - EA 7470, Rennes, France

People generally coordinate their action to be more efficient. However, in some cases, interference between them occur, resulting in an inefficient collaboration. For example, if two volleyball players collide while performing a serve reception, they can both miss the ball. The main goal of this study is to explore the way two persons regulate their actions when performing a cooperative task of ball interception, and how interference between them may occur. Starting face to face, twenty-four participants (twelve teams of two) had to physically intercept balls moving down from the roof to the floor of a virtual room. To this end, they controlled a virtual paddle attached to their hand moving along the anterior-posterior axis. No communication was allowed between participants

Edited by:

Regis Kopper,

University of North Carolina at

Greensboro, United States

Reviewed by:

Ulysses Bernardet,

Aston University, United Kingdom

Alexander Kulik,

Bauhaus-Universität

Weimar, Germany

*Correspondence:

Charles Faure

charles.faure@ens-rennes.fr

Richard Kulpa

richard.kulpa@univ-rennes2.fr

Specialty section:

This article was submitted to

Virtual Environments,

a section of the journal

Frontiers in ICT

Received: 15 March 2019 Accepted: 14 October 2019

Published: 29 October 2019

Citation:

Faure C, Limballe A, Sorel A, Perrin T, Bideau B and Kulpa R (2019) Dyadic Interference Leads to Area of

Uncertainty During Face-to-Face Cooperative Interception Task.

Front. ICT 6:20.

doi: 10.3389/fict.2019.00020 so they had to focus on visual cues to decide if they should perform the interception or leave the partner do it. Participants were immersed in a stereoscopic virtual reality setup that allows the control of the situation and the visual stimuli they perceived, such as ball trajectories and the information available on the partner's motion. Results globally showed participants were often able to intercept balls without collision by dividing the interception space in two equivalent parts. However, an area of uncertainty (where many trials were not intercepted) appeared in the center of the scene, highlighting the presence of interference between participants. The width of this area increased when the situation became more complex (facing a real partner and not a stationary one) and when less information was available (only the paddle and not the partner's avatar). Moreover, participants initiated their interception later when real partner was present and often interpreted balls starting above them as balls they should intercept, even when these balls were in fine intercepted by their partner. Overall, results showed that team coordination here emerges from between-participants interactions and that interference between them depends on task complexity (uncertainty on partner's action and visual information available).

Keywords: virtual reality, interpersonal cooperation, collaborative interactions, ball interception, perceptionaction, team play, team interference, area of uncertainty

\section{INTRODUCTION}

In daily life, people usually interact with others, sometimes by combining their efforts to reach a common goal. However, this cooperation is not always well performed because it implies to concurrently deal with the success of the task and the others' action. Interacting persons may thus have to determine how they must correctly coordinate their actions to be efficient without 
interfering with others. A lot of previous studies were interested in the way people make this coordination. In this work, we are particularly interested in the way interference lead to the creation of an area of uncertainty between participants and how it alters their coordination. This study will be made in the context of a shared task of ball interception.

Interpersonal coordination in joint action tasks has been widely explored in literature. To assess it in a task-directed complementary cooperation task, Richardson et al. (2015) designed a study where participants, divided in teams of two, had to move a computer stimulus back and forth, continuously, between two sets of targets, while avoiding collision with each other. They found that pairs of participants were able to quickly adopt a stable and asymmetric pattern of movement coordination, acting as a team to perform the task with the emergence of dynamic and complementary action behavior. Rigoli et al. (2015) examined the interpersonal coordination that emerged between two participants performing a virtual labyrinth ball-control game and found that the control dynamics of single individuals and pairs were similar, and that pairs performed as well as individuals. Some studies also explored the role of available cues on the coordination in joint action task, and showed importance of visual (Vesper and Richardson, 2014), auditive (Wing et al., 2014), or haptic (Sofianidis et al., 2012) information on movement synchronization. In a recent study by Eils et al. (2017), dyads composed of one Leader and one Follower performed a whole-body joint task involving or not visual and haptic information. They stood on a board and had to guide a virtual ball through a maze toward a virtual hole by jointly shifting their weight on the board. The task had to be performed as fast as possible and three conditions were used: (1) The Follower did not see the Leader nor the maze, (2) The Follower saw the Leader but not the maze, and (3) The Follower saw both the maze and the Leader. Performance was better when visual cues were available, especially when The Follower saw both the maze and the Leader. As a result, two agents are able to coordinate their action and use available information in order to synchronize their movement during a joint action task.

In this paper, we are particularly interested in joint action in a ball interception task. Many authors have looked at the interception of an object (Chardenon et al., 2005; Craig et al., 2006, 2009; Fajen and Warren, 2007; Warren and Fajen, 2008; Ledouit et al., 2013) or a player (Watson et al., 2011; Brault et al., 2012), but only few have focused on the interaction between two or more players. Benerink et al. (2016) studied the interaction between two players in a collaborative pong task: two players had to intercept different ball trajectories on a screen through the use of paddles manually controlled, while avoiding collision with each other. They only had visual feedback on their partner's actions (i.e., they could only see paddles moving on the screen) and authors examined the way players decided who made the interception, and where. Overall interception was good, and collisions between participants were rare. Additionally, they found that decisions regarding who intercepted the ball emerged from interaction and intrinsic cooperation between the two players, in an online manner.
These studies gave interesting results about the way participants coordinate their actions to perform the task together and better than alone. However, it is not always the case. It is indeed not rare to observe partners that interfere with each other, for example in a team sport, when catching a fly ball in baseball, an up and under kick in rugby, or performing a serve-reception in volleyball. And although the ability to coordinate actions as a team is often paramount for succeeding in competitive team sports (Silva et al., 2013), in some cases, the interference results in conflicting situations with an unsuccessful interception or even a collision. The goal of this paper is to look at the interference created by the conflicting situation, and its evolution related to the task complexity. Moreover, we wanted to setup situations with stereoscopic vision, 3D environment and physical involvement in the task. Strong motor coordination and more complex visual cues indeed increase the task difficulty and can thus lead to more conflicting situations.

To propose such complex situations while controlling visual stimuli of the participants, we based our experiment on a virtual reality (VR) setup. Several studies showed the interest of VR to explore interactions and interceptions, for instance in sports situations. Bideau et al. (2010) highlighted that it offers a way to standardize and control situations, and to limit deleterious effects of low-ecological procedures (high degree of subjectivity, lack of immersion, etc.). These observations were corroborated by Vignais et al. (2015) who showed that the performance achieved by players was better in VR compared to video during a ballinterception task in handball. VR has also been shown to be an efficient tool to train interpersonal coordination (e.g., in a team rowing task Varlet et al., 2013). Therefore, we setup a VR-based experiment to control the visual stimuli (ball trajectories and representation of teammate's motion) and to study their influence on the interaction and interference between participants. We allowed the users to be physically involved in the task since it is important to preserve the perception-action loop (Craig, 2013; Farrow, 2013). Moreover, Cañal-Bruland et al. (2010) showed the importance of viewpoint to better perceive a situation involving others: handball goalkeepers were asked to identify whether a penalty-thrower produced a true shot or a deceptive movement, and found better results in the 1st person view condition compared to the 3 rd person view condition. For this reason, we used a 1st person view in our experiment.

Our study thus aims at examining the way two persons interact and interfere when performing a cooperative task of ball interception, without collision and only based on visual cues. To explore this interpersonal cooperation, we designed a study where participants had to physically intercept a moving ball, through the use of a paddle held in their hand, moving along a shared axis, so that interception could only be successfully performed by one participant. As Benerink et al. (2016) found in their study, we expect participants to adopt a strong cooperation pattern of interception. We aim to identify and quantify situations when team cooperation is less efficient and thus when interference appears between teammates. In particular, we would like to explore if team organization depends on ball trajectory and on information available on the partner and her/his action (i.e., the movement of the paddle or the upper body). 


\section{MATERIALS AND METHODS}

\subsection{Participants}

Twenty-four right-handed participants (sixteen males and eight females), aged $32.9 \pm 8.9$ years (mean $\pm \mathrm{SD}$ ), took part in this study. Mean height was $173.6 \pm 5.8 \mathrm{~cm}$. None of the participants had injury at the moment the experiment took place, they did not know each other, and all of them gave their written consent to take part in this study. No participant experienced motion sickness or got injured. The study was led with respect to the Declaration of Helsinki and participants were all informed they could withdraw from the study at any time, and none of them did.

\subsection{Experimental Set-Up}

The experiment took place in a dark sports hall, large enough to avoid collision with the physical world. Figure 1 presents the experimental setup and the virtual environment used, and Figure 2 a sample of trials performed by a representative participant for each condition (see also Supplemental Video 1). The virtual environment consisted in a simple and basic room (L*1*h: $6^{*} 4^{*} 4 \mathrm{~m}$ ). The environment was built with Unity software (Version 5.6.6). The origin of the scene was placed at the center of the scene.

To interact with the virtual world, participants used their dominant hand (all participants were right-handed) to grab a virtual paddle (dimensions $\left(\mathrm{w}^{*} \mathrm{l}^{*} \mathrm{~h}\right): 40^{*} 25^{*} 10 \mathrm{~cm}$ ), placed 1.3 $\mathrm{m}$ above ground, and were free to physically move in the entire space to try and intercept balls with it. The paddle could only be displaced along the anterior-posterior axis (now referred to as the $\mathrm{X}$-axis) to intercept balls moving in the sagittal plane. This control of the paddle displacement ensured that participant could not get around or above their partner's paddle during ball interception. In order to avoid physical collision between participants, each one was located in his/her own physical environment but performed the task in a shared virtual environment. Synchronization between the two Head-Mounted Display (HMD, model: HTC Vive, Field of View: $110^{\circ}$, image resolution: $2160 * 1200$ pixels) devices was made through a local network leading to a latency of about $33 \mathrm{~ms}$, which is close to the HTC Vive system end-to-end latency of about $22 \mathrm{~ms}$ (Niehorster et al., 2017), and even better than the "low latency threshold" of 50 ms defined by Meehan et al. (2003).

\subsection{Task}

The task comprised four different conditions, in which participants were asked to intercept with the virtual paddle balls moving down from the roof to the floor of the $3 \mathrm{D}$ immersive room with a linear trajectory. During the first condition, participants were alone to intercept balls (Figure 2A, now referred as AL condition). This condition was used as the reference. In the second condition, participants were assisted by a stationary partner: a long paddle (length: $1.75 \mathrm{~m}$ ) that could also intercept balls (Figure 2B, now referred as SP condition). Length and position of the stationary paddle were calculated so it could intercept balls moving toward the farthest ending points (probability of ball hitting the stationary paddle $=0.44$ ), from the participant's viewpoint, based on data from pilot study (Faure et al., 2017). The third and fourth conditions were performed by teams of two real participants who had to intercept the ball as a team. In the third condition, participants could only see the movements of their partner's paddle (Figure 2C, now referred as PP condition), whereas in the fourth condition, they could see both the avatar of the partner and her/his paddle (Figure 2D, now referred as AP condition). The avatar was represented as a simple stick man (head + upper limbs). The head and the hands of the avatar were animated with the headset and the controllers movements. Both head and hands movements were mapped on the avatar. Arms of the avatar were reconstructed with the use of an inverse kinematics script. This choice of a simple avatar was made for several reasons: (1) as we mainly wanted to study global interaction and perception of ball trajectories, we only needed participants to perceive global partner's displacement; (2) absence of important behavioral cues such as gaze and facial expression can partly be compensated, with participants shifting their attentions to other behavioral channels (Roth et al., 2016); and (3) the Level Of Details (LOD) do not influence perception of movement (Vignais et al., 2009, 2010), and it has been suggested that a reduction in stimulus information may facilitate the perception of a movement (Lintern et al., 1990; Farrow, 2013).

In all conditions, participants started the experiment at one extremity of the virtual world. It means that, for PP and AP conditions, participants were facing each other at a distance of 4 $\mathrm{m}$, which represented the total length of the experimental setup. In SP, a stationary partner was placed in front of them. The virtual environment was designed so they could see both ball and their teammate performing the task. From experimenter's viewpoint, participant at the right was referred as $P_{1}$, and participant at the left was referred as $P_{2}$. Participants kept the same side when performing $\mathrm{PP}$ condition and AP condition. In our study, the task was performed alone (AL condition) or in collaboration with a partner that can be virtual and static (SP) or real and moving (PP and AP).

\subsection{Procedure}

Before each trial, participants had to move to their starting position, materialized by a virtual red or blue cross, located -2 $\mathrm{m}$ or $+2 \mathrm{~m}$ from the center of the scene, on the X-axis. When participants were ready, an external experimenter started the trial. A long trapdoor, located in the roof, immediately opened, and a ball moved downward with a velocity of $2.5 \mathrm{~m} / \mathrm{s}$, resulting in trials where participants had between 1.1 and $1.6 \mathrm{~s}$ to intercept the ball. As perception of trajectories is greater when using a colored ball compared to a plain white ball (Lenoir et al., 2005), a high-contrast virtual blue-yellow-white ball (circumference: 65 $\mathrm{cm}$ ) was designed and used for the study.

Based on protocol by Benerink et al. (2016), ball trajectories were set up with the use of five departure points (located at a height of $4 \mathrm{~m}$ ) and five arrival points (located at a height of $1.3 \mathrm{~m}$ ). This choice of departure and arrival points' height was made so participants can see both ball and partner paddle at any time in their field of view. Departure points were separated by $0.8 \mathrm{~m}$, as well as arrival points (both at $\mathrm{X}=$ $-1.6,-0.8,0,+0.8$, and $+1.6 \mathrm{~m})$. The combination of departure and arrival points resulted in the construction of 25 standard 

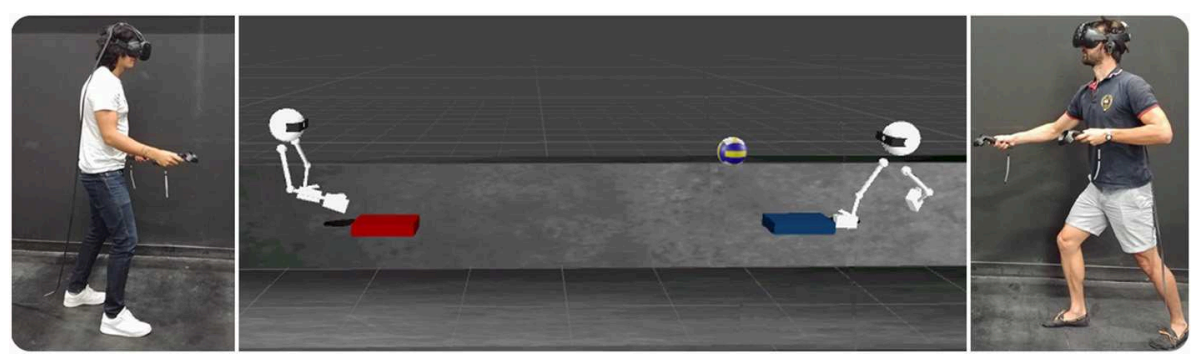

FIGURE 1 | Experimental Virtual Reality-based setup used to study the full-body cooperation between two participants in a ball interception task. Both participants gave their written informed consent for the use of photographs.
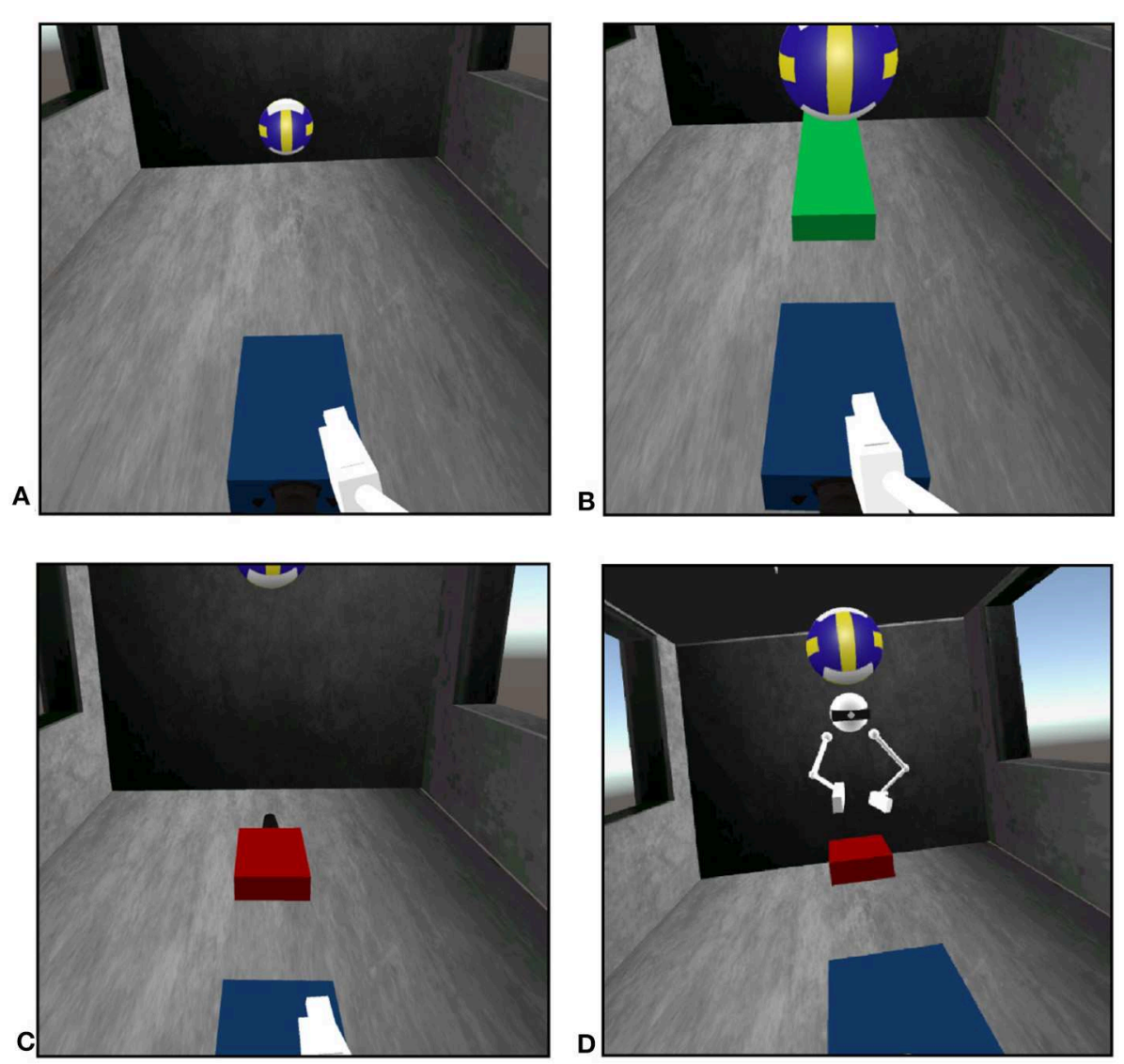

FIGURE 2 | Task of interception-participant's viewpoint. (A) Alone Condition (AL): participant performed the task alone. (B) Stationary Partner Condition (SP): participant performed the task assisted by a stationary partner. (C) Paddle Partner Condition (PP): participant performed the task with a real partner, but could only see her/his paddle. (D) Avatar Partner Condition (AP): participant performed the task with a real partner, and could see her/his paddle as well as her/his avatar.

trajectories, for a total of 100 trials to intercept for each subject. It is important to note that, for PP and AP conditions, participants performed the task by teams of two. It implies that each team performed 50 trials in AL and SP conditions and only 25 trials in $\mathrm{PP}$ and AP conditions (see Figure 3). As a result, the 12 teams performed 600 trials in AL (25 trials*24 participants), 600 trials in SP (25 trials*24 participants), 300 trials in PP (25 trials* 12 teams) and 300 trials in AP (25 trials*12 teams).
Moreover, in order to avoid familiarization with departure and arrival points, a random distance comprised between -0.4 and $0.4 \mathrm{~m}$ was added to both departure and arrival points, for each trial. Balls could thus appear anywhere along the trapdoor, and fall anywhere on the arrival area, with no change of trajectory angle. The five departure and five arrival points were invisible to participants.

Before the experiment started, participants were asked to move in the virtual room and to perform 10 trials so they get 


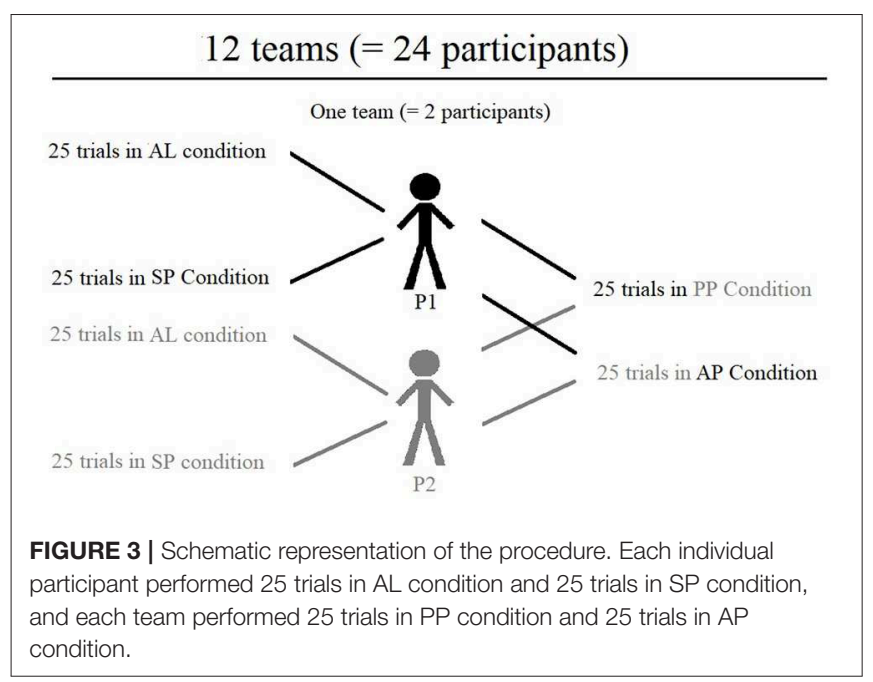

familiar with the task and the virtual environment. Moreover, when assisted by a partner (stationary or real), they had to voluntarily collide it once to understand that it is considered as a failure. None of these trials was recorded. Participants all started the experiment performing the AL condition, i.e., the condition where they were alone to intercept balls. Then, they performed the three other conditions randomly. In order to avoid fatigue (due to experiment or prolonged use of HMD device), a rest-period (minimum of $5 \mathrm{~min}$ ) was given between conditions.

A trial was considered successful when the ball was intercepted with the paddle. A trial was considered unsuccessful when none of the participants intercepted the ball or when a collision between paddles occurred. If a ball was intercepted but a collision occurred after, the associated trial was considered unsuccessful. A message was displayed at the end of each trial to inform participants whether the trial was successful or not. When a collision occurred, a message was displayed to inform participants that they collided and that the trial was unsuccessful. At the end of each trial, participants were asked to return to their starting points, and the experimenter launched a new trial. For PP and AP, i.e., conditions where teams of two participants were involved, no verbal communication was allowed before or during the experiment. Eliminating verbal communication was implemented to ensure that the decision to move was based solely on the partner's movements.

\subsection{Dependent Variables and Analysis}

Players' head and hand displacements were recorded through the displacement of HTC Vive headset and controllers. As the setting of the scale of the virtual environment was built to match exactly the scale of the real environment, displacement was the same in virtual and physical worlds.

Interception score was calculated as the percentage of balls intercepted among the total number of trials, for each condition. Number and position of virtual collisions per condition were collected.

In a preliminary study, we found that, when participants were assisted with a stationary partner, they missed trials they were able to intercept when they performed the task alone (Faure et al., 2017): the presence of a stationary partner led participants to be less effective when intercepting balls close to the stationary partner, although overall interception was better. To assess if similar results were found in the present study, when trials were missed in AL and SP conditions, we computed the distance to cover, i.e., the distance between paddle starting position and ball arrival position, for the balls that had to be intercepted by the participant. It means that to evaluate the distance to cover, we excluded from AL results all the missed balls that ended in the area where stationary paddle was positioned in SP condition. This distance could thus be seen as the distance that participant should have covered to intercept the ball: the smaller this mean value, the closer to participant's starting position the missed balls ended. This way, we evaluated if participants missed trials in SP they were able to intercept in $\mathrm{AL}$, as previously found in the pilot study. More generally, we explored whether or not interference between participants could appear during collaborative conditions, and how task complexity and available visual cues could influence this interference.

During a trial, initiation of movement was defined as the first moment when participant's hand velocity reached the threshold of $0.5 \mathrm{~m} / \mathrm{s}$ with a minimal displacement of $10 \mathrm{~cm}$ on $\mathrm{X}$-axis. When the ball was intercepted, the location of the interception was computed, and when not, the ending position of ball was collected.

Data post-processing, variables analysis and statistics were made with $\mathrm{R}$ software ( $\mathrm{R}$ Core-Team, 2016), and significance level was set to 0.05 . Normality of data was tested with ShapiroWilk test. The effect size (ES) was calculated to evaluate the size of statistical effects observed, and computed using partial eta squared $\left(\eta_{p}^{2}\right)$ for ANOVA's (regarding Cohen's scale; small effect: $0.01<\eta_{p}^{2}<0.06$, medium effect: $0.06<\eta_{p}^{2}<0.14$, and large effect: $\eta_{p}^{2}>0.14$ ) and Cohen's $d$ for pairwise comparisons (according to Cohen's scale; small effect: $0.2<d<0.5$, medium effect: $0.5<d<$ 0.8 , and large effect: $d>0.8$ ).

\section{RESULTS}

\subsection{Interception and Collision}

First of all, to ensure there was no effect of gender on performance, we conducted a mixed two-way ANOVA on interception performance with gender (Male and Female) as a between-participant factor, and condition (AL, SP, PP, and AP) as a within-participant factor. Results showed no difference in performance between males and females, for all conditions $\left[\eta_{p}^{2}\right.$ $=0.02, p=0.71]$.

Overall interception score during the experiment was calculated for each condition (Table 1). When participants were alone (AL), they intercepted $53.00 \pm 11.20 \%$ of balls. When they were assisted by a stationary partner (SP), global interception score was higher $(89.33 \pm 6.46 \%)$. When they performed the task with a real partner (PP and AP), performance was also good $(83.00 \pm 7.84 \%$ and $86.33 \pm 9.86 \%$, respectively). Nonparametric Kruskal-Wallis test revealed a significant effect of condition on performance $\left(\chi^{2}=47.35, d f=3, p<0.001\right)$, and 
TABLE 1 | Global interception score and collision during experiment.

\begin{tabular}{lcc}
\hline Condition & $\begin{array}{c}\text { Overall interception } \\
\text { score }(\% \pm \boldsymbol{S D})\end{array}$ & $\begin{array}{c}\text { Collisions (number } \\
\text { and \% of trials) }\end{array}$ \\
\hline AL condition & $53.00 \pm 11.20$ & - \\
SP condition & $89.33 \pm 6.46^{\star \star \star}$ & $3(0.5 \%)$ \\
PP condition & $83.00 \pm 7.84^{\star \star \star}$ & $7(2.33 \%)$ \\
AP condition & $86.33 \pm 9.86^{\star \star \star}$ & $6(2 \%)$
\end{tabular}

***Significantly different from $A L(P<0.001)$.

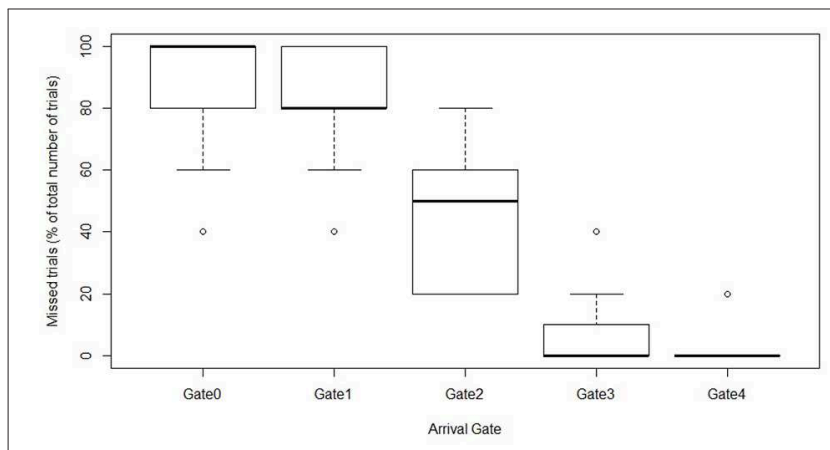

FIGURE 4 | Mean interception score (in \%) per subject, related to arrival gates, when participants were alone (AL Condition).

Pairwise comparisons using Wilcoxon rank sum test (Bonferroni correction) revealed performance was significantly lower during $\mathrm{AL}$ compared to SP, PP, and AP $(d>0.8, p<0.001)$. Moreover, there was no difference in performance between SP, PP, and AP.

Collisions with partner were very rare (Table 1), whether the partner was stationary (SP, 3 collisions) or real (PP and AP, 7 and 6 collisions, respectively).

To ensure position and length of partner paddle were correctly set up, we looked at the number of unsuccessful trials per subject in $\mathrm{AL}$ condition, as a function of the five ball arrival positions (Figure 4). As expected, when arrival position was far from participants (i.e., Gate0 and Gate1), trials were mainly unsuccessful $(86.67 \pm 19.69 \%$ and $83.33 \pm 18.74 \%$ of missed trials, respectively). On the contrary, when ball arrival position was close to participants (i.e., Gate3 and Gate4), trials were mainly successful $(8.33 \pm 15.86 \%$ and $1.67 \pm 5.77 \%$ of missed trials, respectively). Finally, when ball arrival position was the middle one, participants missed $45 \pm 21.11 \%$ of trials. This observation is in accordance with the choice, in SP condition, to place the stationary paddle so it could intercept balls that participants had difficulties to intercept in first condition. Moreover, to ensure participants correctly intercepted balls arriving close to their departure position (i.e., Gate3 and Gate4) in all conditions, we performed a mixed two-way ANOVA on interception percentage with arrival gate (Gate3 and Gate4) as a between-participant factor and condition (AL, SP, PP and AP) as a within-participant factor. Results showed no difference in performance, for all conditions $\left[\eta_{p}^{2}=0.02, p=0.66\right]$ : participants correctly intercepted the closest balls, for all conditions.

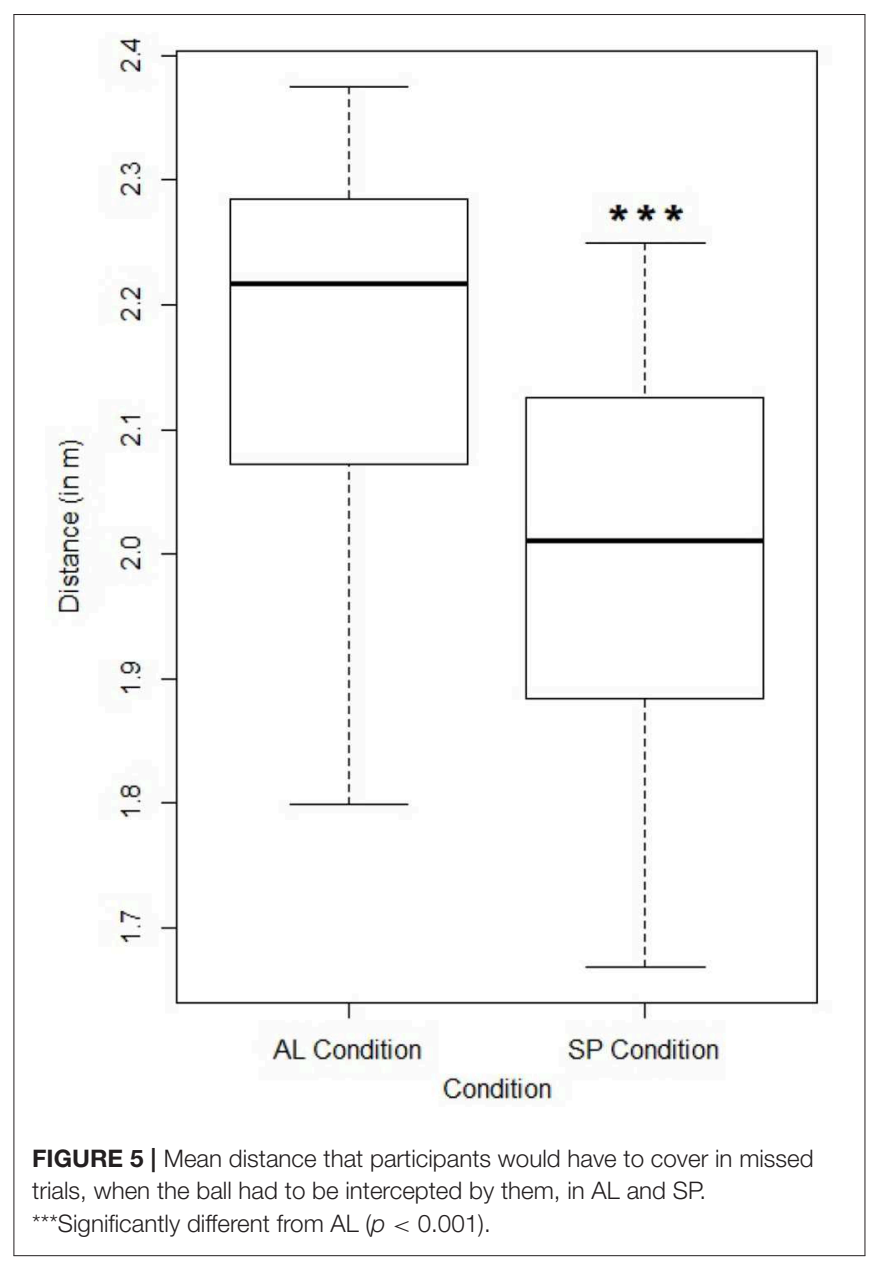

\subsection{Area of Uncertainty}

Analysis of distance to cover revealed that this distance in missed trials was smaller in SP $(1.99 \pm 0.16 \mathrm{~m})$ than in AL condition $(2.18 \pm 0.15 \mathrm{~m})$ and this result was significant [paired samples $t$-test: $t_{(49)}=4.45, p<0.001$ ] (see Figure 5). To confirm this observation, we explored the interception score as a function of ball arrival position (Figure 6). Interceptions performed by participants $\left(P_{1}\right)$ are plotted in green, and interceptions performed by the stationary partner $\left(\right.$ Stationary $_{P}$ ) are plotted in blue. Similarly, ball arrival positions of unsuccessful trials are represented by dots: red for collisions and yellow for missed trials. When the ball was missed or when a collision occurred, the final ball position was often close to the stationary paddle. As a result, this suggests participants' ability to coordinate interception with a stationary partner was very good (high interception score), but performance was less effective when ball arrival point was close to the stationary partner paddle (this area will now be referred to as the area of uncertainty). Moreover, only few interceptions were performed by participants in this area, although interception domains were well defined. Indeed, as partner paddle was not able to move, its interception domain was fixed. To go further, we used a logit link function (Hosmer et al., 2000) and plotted the logistic curve representing the probability that $P_{1}$ (probability 


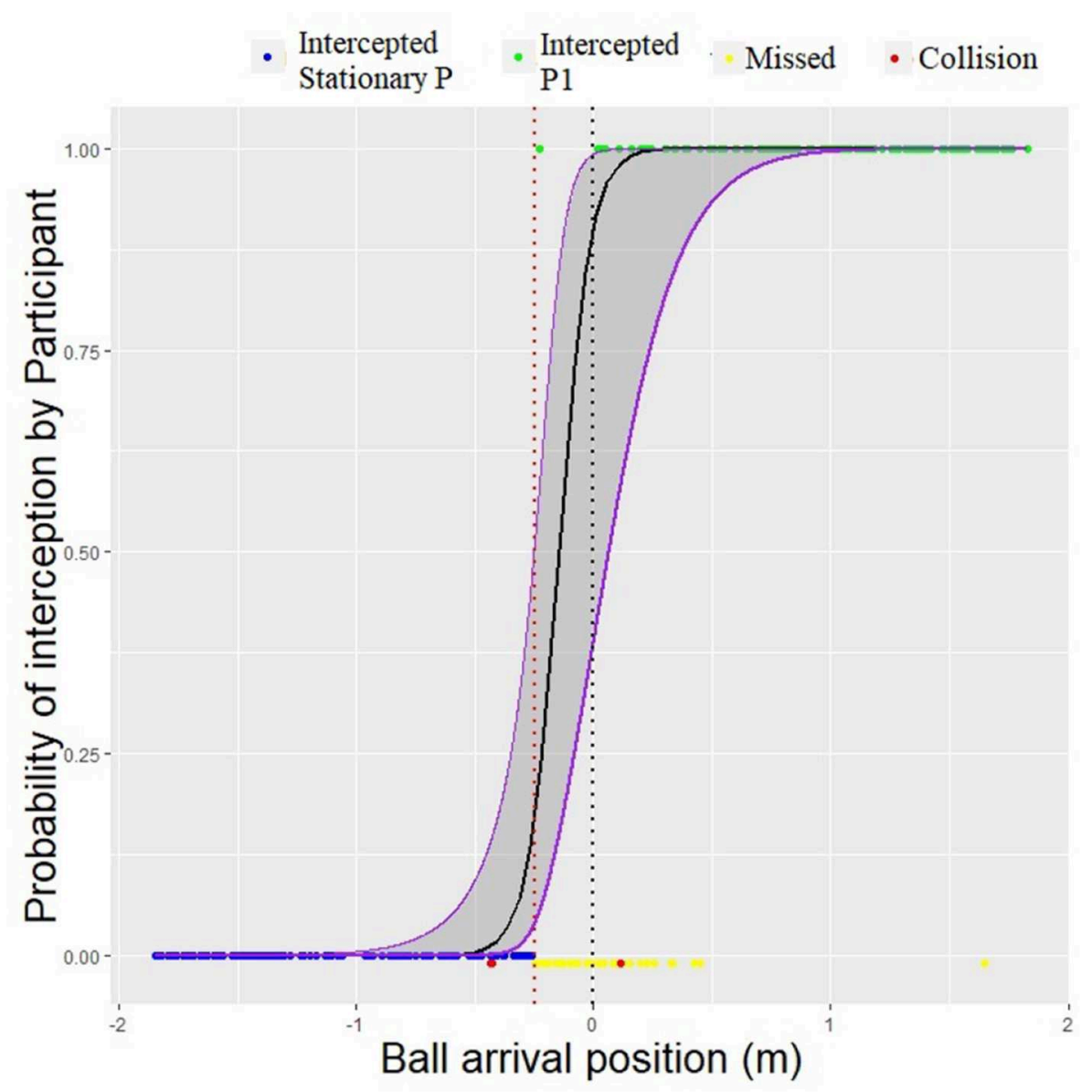

FIGURE 6 | Graphical summary of interception performance as a function of ball arrival position (SP Condition). Green dots represent arrival positions of balls intercepted by Participant, and blue dots represent arrival positions of balls intercepted by Stationary Partner. Ball arrival positions of unsuccessful trials are indicated by yellow dots (missed trial) and red dots (collision). Vertical dashed black line indicates the center of the X-axis, and vertical dashed red line indicates the limit of the stationary paddle. Black curve represent the logistic curve related to the probability that $P_{1}(P=1)$ or Stationary $P(P=0)$ intercept the ball, as a function of ball arrival position. Purple curves represent the 95\% Confidence Interval.

$P=1$ ) or Stationary $P$ (probability $P=0$ ) intercepts the ball, as a function of ball arrival position. If interception domains were perfectly defined, separation should be complete, i.e., it could be possible to find a "vector $\alpha$ that correctly allocates all observations to their group" (Albert and Anderson, 1984). Consequently, in our specific case, probability $\mathrm{P}$ should be equal to 0 for balls ending in the stationary partner, and equal to 1 everywhere else. To quantify the difference between this theoretical aspect and the real results, we looked at the Median Effective Level (MEL) (Bewick et al., 2005), that is the position on X-axis at which the two possible outcomes are equally likely, i.e., when $\mathrm{P}\left(P_{1}\right)=$ $\mathrm{P}\left(\right.$ Stationary $\left._{P}\right)=0.5$. This value represents the distance between theoretical boundary limit (in SP condition, the proximal edge of the partner paddle) and real boundary. MEL in SP was 10.5 $\mathrm{cm}$, indicating a deviation of the boundary by $10.5 \mathrm{~cm}$ on the right, i.e., toward the real participant. To better quantify the area of uncertainty previously defined, the steepness of the slopes of the logistic curve was used, and in particular, we looked at the amount of overlap, defined by Cox and Snell (1989) as the distance between the $5 \%$ and $95 \%$ points of this curve. We made the choice to adopt the same approach as Benerink et al. (2016) in order to better discuss our results on overlap. Overlap in SP was of $38.5 \mathrm{~cm}$ and represents a mathematical quantification of the area of uncertainty. This means that, in this $38.5 \mathrm{~cm}$-wide area, participants had difficulties to judge whether or not they should intercept the ball.

In order to examine a potential effect of side in PP and AP, i.e., a potential difference in performance between $P_{1}$ and $P_{2}$, we performed a mixed two-way ANOVA on interception scores with side $\left(P_{1}\right.$ and $\left.P_{2}\right)$ as a between-participant factor and condition (PP and AP) as a within-participant factor. Results showed no difference in performance between $P_{1}$ and $P_{2}$, in both conditions $\left[\eta_{p}^{2}=0.0007, p=0.86\right]$.

Using similar logistic regression analysis as before, we examined whether or not the area of uncertainty was influenced by the interaction between two real partners. Figures 7, 8 present graphical summary of interception as a function of ball arrival position, for PP and AP, respectively. In PP, MEL value was -12.2 


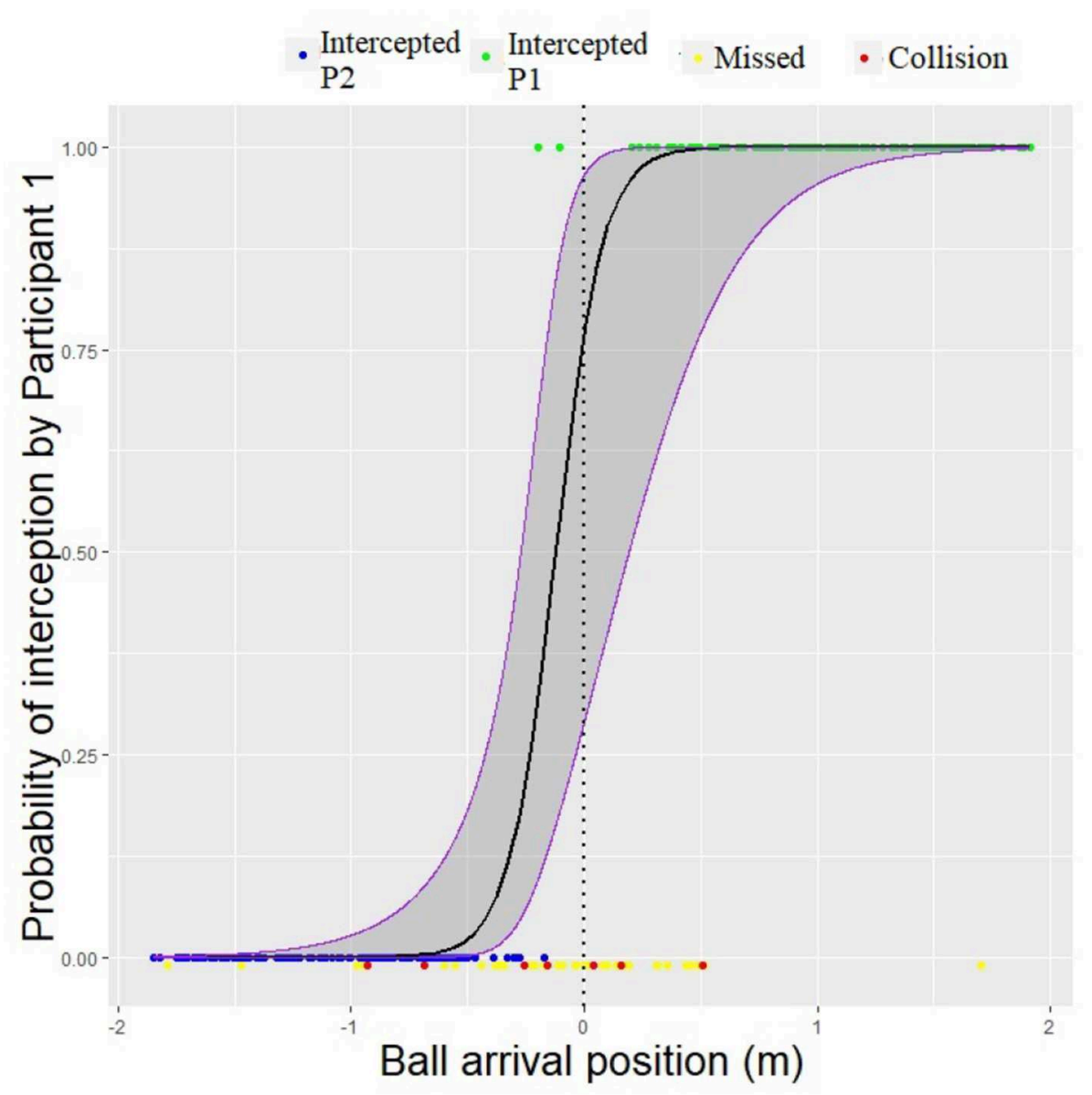

FIGURE 7 | Graphical summary of interception performance as a function of ball arrival position (PP Condition). Green dots represent arrival positions of balls intercepted by Participant 1, and blue dots represent arrival positions of balls intercepted by Participant 2. Ball arrival positions of unsuccessful trials are indicated by yellow dots (missed trial) and red dots (collision). Vertical dashed black line indicates the center of the X-axis. Black curve represent the logistic curve related to the probability that $P_{1}(P=1)$ or $P_{2}(P=0)$ intercept the ball, as a function of ball arrival position. Purple curves represent the $95 \%$ Confidence Interval.

$\mathrm{cm}$, and overlap was $59.4 \mathrm{~cm}$. In AP, MEL value was $-1.2 \mathrm{~cm}$ and overlap was $49.1 \mathrm{~cm}$. Moreover, to support the idea that area of uncertainty was located around the theoretical boundary line, we found that in unsuccessful trials, when no movement of interception was initiated by any of the participants, final arrival gate of the ball was often the middle one, in SP $(86.27 \%$ of missed trials), PP (66.67\% of missed trials) and AP $(82.35 \%$ of missed trials).

\subsection{Movement Initiation Time}

To explore the influence of conditions on participants' kinematics of movement, we examined movement the initiation time, for all trials. Table 2 shows movement was initiated later when a real partner was present compared to when participants were alone, for overall trials. A repeated-measures one-way ANOVA confirmed this influence of condition on initiation time $\left[\eta_{p}^{2}=\right.$ 0.10, $p<0.05$ ] and Post-hoc Tukey HSD revealed initiation of movement was significantly higher in $\mathrm{PP}$ and AP compared to $\mathrm{AL}$ condition $[p<0.05]$. Interestingly, when looking only at intercepted trials, no difference between conditions was found (one-way ANOVA: $\eta_{p}^{2}=0.01, p=0.33$ ). On the other hand, when focusing on unsuccessful trials, repeated-measures oneway ANOVA revealed influence of condition on initiation time $\left[\eta_{p}^{2}=0.15, p<0.001\right]$, and Post-hoc Tukey HSD confirmed this observation with significantly higher initiation of movement in PP and AP compared to AL and SP conditions $(p<0.05)$. Finally, there was no difference in initiation of movement between unsuccessful and successful trials, for all conditions $\left[\eta_{p}^{2}=0.13\right.$, $p=0.11]$.

\subsection{Abandoned Movement of Interception}

In $\mathrm{PP}$ and AP conditions, interception could only be performed by one of the two participants, but both participants could initiate a movement of interception. In that case, it means that one of them had to stop the movement to let the partner intercept the ball. Regarding this aspect, we explored whether or not departure or arrival gate influenced this abandoned interception movement. To be considered as an abandoned interception movement, a trial had to encounter three conditions: (1) the participant had a minimal displacement of $50 \mathrm{~cm}$ on 


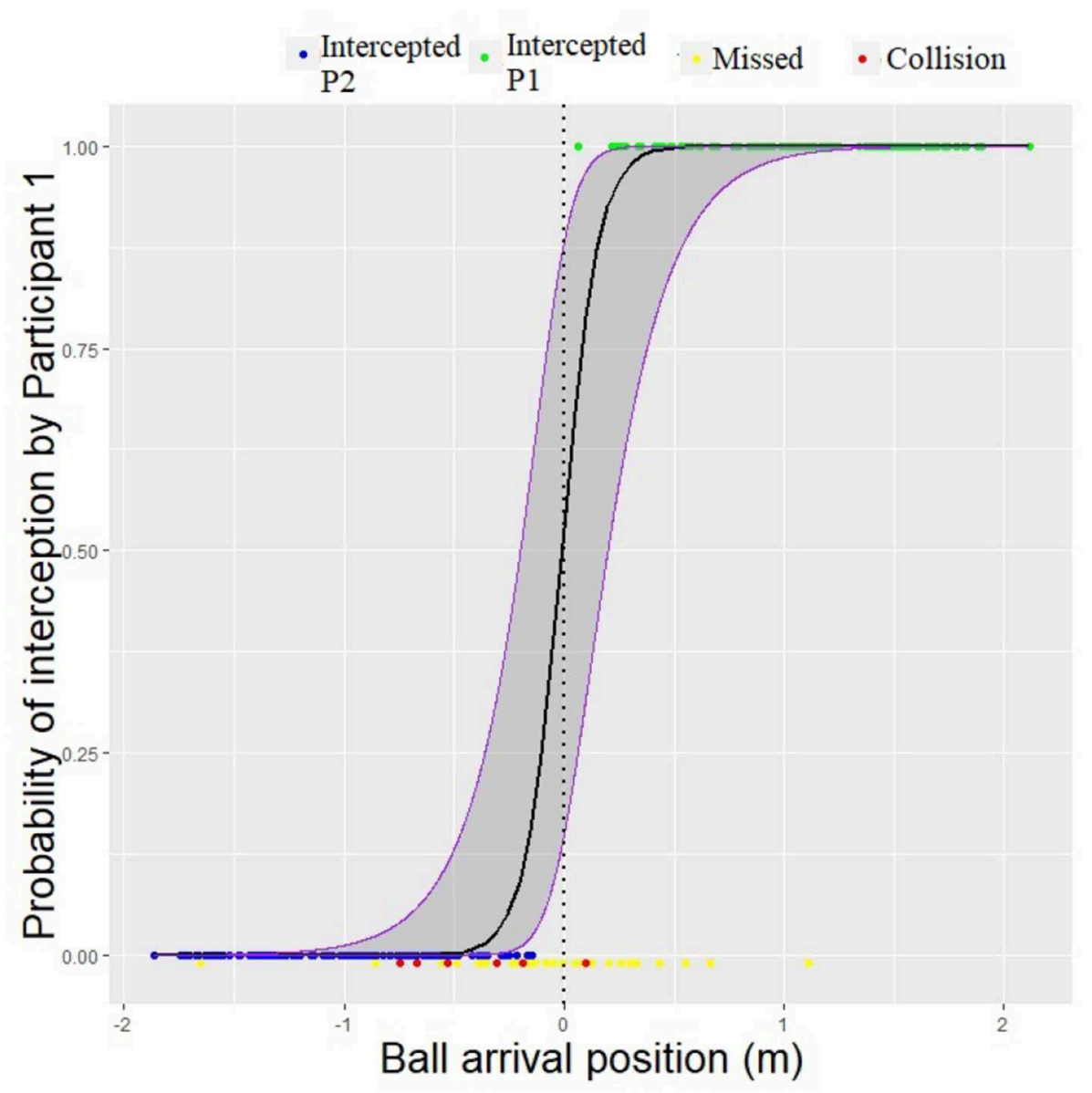

FIGURE 8 | Graphical summary of interception performance as a function of ball arrival position (AP Condition). Green dots represent arrival positions of balls intercepted by Participant 1, and blue dots represent arrival positions of balls intercepted by Participant 2. Ball arrival positions of unsuccessful trials are indicated by yellow dots (missed trial) and red dots (collision). Vertical dashed black line indicates the center of the X-axis. Black curve represent the logistic curve related to the probability that $P_{1}(P=1)$ or $P_{2}(P=0)$ intercept the ball, as a function of ball arrival position. Purple curves represent the $95 \%$ Confidence Interval.

the X-axis, (2) velocity of participant's paddle became null or negative during the trial, and (3) interception was in fine performed by the partner. We found no influence of arrival gate $\left[\eta_{p}^{2}<0.01, p=0.20\right]$ but a significant influence of departure gate $\left[\eta_{p}^{2}=0.04, p<0.01\right]$ on abandoned interception movement in PP and AP. Post-hoc Tukey HSD revealed that the closer the departure gate was, the later the interception was abandoned.

\section{DISCUSSION}

\subsection{Interception and Collision}

We first found that interception performance was good, with higher scores in SP, PP, and AP compared to AL: overall performance was thus better in collaborative conditions. This seems logical since participants only had difficulties in intercepting farthest balls in AL (see Figure 4), i.e., balls that were intercepted by their partner in SP, PP, and AP. Second finding highlighted good participants' ability to efficiently coordinate their action, with very few collisions with partner paddle (16 collisions over the total 1,200 trials performed in collaborative conditions).

\subsection{Area of Uncertainty}

However, when looking deeper into the results, distance to cover revealed that in SP, participants missed many trials they were able to intercept when they were alone (AL). As a consequence, when assisted by a stationary partner, participants were less effective in intercepting balls ending close to the partner. Literature showed that virtual balls without spin can be effectively intercepted (Craig et al., 2006, 2009, 2011; Dessing and Craig, 2010; Bootsma et al., 2016), a result also observed in our study. Then, it suggests that errors made by participants in SP condition may be due to an error in judgment of ball trajectory: participants did not intend to intercept the ball when they thought it would end on the partner paddle, even if it was not in fine the case. In this context, the area of uncertainty reflects the zone where participants had difficulties to judge ball trajectory. 
TABLE 2 | Mean movement initiation time of participants in all conditions, for overall, intercepted and unsuccessful trials.

\begin{tabular}{llll}
\hline Condition & $\begin{array}{l}\text { Movement } \\
\text { initiation time for } \\
\text { overall trials (in } \\
\text { ms, mean } \pm \text { SD) }\end{array}$ & $\begin{array}{l}\text { Movement } \\
\text { initiation time for } \\
\text { successful trials (in } \\
\text { ms, mean } \pm \text { SD) }\end{array}$ & $\begin{array}{l}\text { Movement initiation } \\
\text { time for } \\
\text { unsuccessful trials } \\
\text { (in ms, mean } \pm \text { SD) }\end{array}$ \\
\hline AL condition & $602 \pm 127$ & $617 \pm 122$ & $524 \pm 127$ \\
SP condition & $632 \pm 152$ & $641 \pm 150$ & $573 \pm 77$ \\
PP condition & $650 \pm 150^{\star}$ & $651 \pm 153$ & $655 \pm 138^{\star \dagger}$ \\
AP condition & $654 \pm 141^{*}$ & $652 \pm 141$ & $664 \pm 144^{\star \dagger}$
\end{tabular}

*Significantly different from $A L(p<0.05)$.

${ }^{\dagger}$ Significantly different from $S P(p<0.05)$.

To better explore this idea, we looked at who intercepted the ball and where, in SP condition. If division of interception space looked pretty well defined (Figure 6), with participants intercepting closest balls and stationary partner intercepting farthest ones (from participant's viewpoint), we could see that most of the unsuccessful trials occurred when ball arrival position was close to the stationary partner's paddle. The area of uncertainty quantified by the logistic regression had a width of $38.5 \mathrm{~cm}$ in our SP condition. However, MEL revealed that the area was centered $10.5 \mathrm{~cm}$ far from the partner paddle's proximal edge, leading to a logical $19.25 \mathrm{~cm}$-wide area at each side of the MEL. The area of uncertainty is thus partly located in the partner paddle's area of interception which is not possible since the partner paddle is stationary and always intercepts balls in the area it covers. The model of logistic regression was then corrected and revealed a $29.75 \mathrm{~cm}$-wide area of uncertainty, starting at the proximal edge of stationary partner paddle. We then tried to identify how this area may be influenced by the visual information available on the real partner. In PP and AP conditions, division of interception space was also pretty well defined (Figures 7, 8), with $P_{1}$ (resp. $P_{2}$ ) intercepting the majority of balls arriving on the positive half (resp. the negative half). Logistic regression was performed and revealed that area of uncertainty was of $59.4 \mathrm{~cm}$-wide and $49.1 \mathrm{~cm}$-wide in PP and $\mathrm{AP}$, respectively. A cooperative interception task with a partner thus created interference between participants and highlighted the apparition of an area of uncertainty, whether the partner was real or not.

Additionally, uncertainty increased when participants performed the task with a real partner compared to a stationary partner. This seems logical because presence of a real partner implied unpredictable movements, contrary to stationary partner. Finally, area of uncertainty was wider when participants had visual cues available only on partner's resulting action compared to partner's action and movement (PP vs. AP). This suggests that participants got information coming from partner's upper limbs and head in order to better organize their interception and perform more efficiently. Interference between participants thus seemed to be influenced by the task complexity (moving vs. stationary partner) and the available visual cues (partner + paddle vs. paddle only).

Moreover, trials where no movement was initiated by any of the participants mainly occurred when ball arrival position was near the center of the scene, meaning that both participants thought the interception should be performed by the other. This result is not in accordance with Benerink et al. (2016), who found that when ball arrival position was close to the center of the scene, there was very few trials where none of the participants initiated a movement. This difference may be due to the fact that, in our situation, participants had to physically intercept the ball. This difference may confirm that, in order to maintain the perceptionaction loop (Craig, 2013; Farrow, 2013), getting physically involved in the task is important, and results can be influenced by this physical involvement. Moreover, physical interception requires physical involvement, and so, avoiding virtual collision when movement is initiated may be more difficult in our study than in Benerink's one. As a result, we could think that when they had a doubt about the final ball ending position, participants in our study chose to rely more on partner than on their own action.

\subsection{Movement Initiation Time}

Looking at kinematics of participants, we found that movement of interception was initiated later when two real participants performed the task ( $\mathrm{PP}$ and AP conditions, see Table 2), compared to the situation when they performed the task alone ( $\mathrm{AL}$ condition). On the other hand, no influence of available information was found on initiation time when two real participants were present (i.e., no difference was found between PP and AP). It seems that participants waited longer before initiating a movement only when they were assisted by a real partner (compared to $\mathrm{AL}$ ). This difference may be due to various elements. First, the uncertainty of the situation added an phase lag before movement is initiated. This is in accordance with the study by Richardson et al. (2015) on behavioral dynamics of an interpersonal collision-avoidance task. Authors indeed showed that participants quickly adopted a stable and asymmetric pattern of movement coordination, but also observed the apparition of an observation phase between participants, as it may also be the case in the present study. It suggests that visual cues of the partner's action are used by participants before they choose and perform their own action. Second, since participants are taking information on the partner's action, the task is more complex than in AL condition. Their reaction time is thus longer since it depends on the task complexity (Darbutas et al., 2013). For instance, Laszlo and Livesey (1977) found that when movements were not goal-directed, reaction times were $100 \mathrm{~ms}$ shorter than when movements were goal-directed, with strict accuracy demands. Third, Benerink et al. (2016) suggested that the decision of who intercepts the ball is not only based on a simple geometrybased division of space, but would also rely on an interaction between participants, when the situation involves more than one participant. Orban de Xivry et al. (2017) also explained that the brain can adapt to start a movement and modify it later, based on new incoming information. This requires participants to adapt to both environment and partner, by modifying their own action of interception online. 


\subsection{Abandoned Movement of Interception}

In our study, although a trial is deemed successful in PP and AP when only one participant intercepts the ball, both participants usually initiate a movement of interception. In that case, one has to give up their own movement in order to avoid collision with the partner. To better understand the influence of task complexity on participants' action, as well as the online coordination, we looked at trials with abandoned interception movement (see details in Results section). We found that departure gate influenced kinematics of interception of participants: the farther the departure gate, the earlier the movement is given up. Interestingly, it seems that participants often interpreted a ball starting close to them as a ball they had to intercept, although the ball finally arrived on the opposite side and was intercepted by the partner. However, they were able to give up their interception movement, based on new incoming information during the trial. Additionally, when focusing only on missed trials, we found that initiation time was also higher in PP and AP compared to AL. Indeed, in AL, unsuccessful trials may be attributed to task difficulty (i.e., difficulty to cover distance toward ball when it arrives on Gate0 and Gate1) whereas in $\mathrm{PP}$ and AP, unsuccessful trials may be attributed to both time to initiate a movement (in missed trials, initiation was often too late to be able to intercept the ball) and arrival position of ball (most of balls in missed trials ended near the center of the scene).

\subsection{General Discussion}

Overall, our results indicate that team coordination emerges from between-participants interactions, and interference between them depends on task complexity (uncertainty on partner's action and visual information available). However, when pairs of real teammates performed the task, we found no influence of available visual cues on the initiation time of interception.

In the dynamic approach of team coordination, inspired by concepts from ecological psychology (Gibson, 1979; Cooke et al., 2013), team level and team performance are driven by interactions between individuals, and real-time coordination. In this context, team efficiency relies on participants' ability to interact and self-regulate their own actions, according to ongoing information available from environment, task and partner, that is, according to shared affordances (Silva et al., 2013). Team interaction can thus be manipulated in order to increase team effectiveness (Gorman, 2014). As an example, players in volleyball often perform a large number of serve reception and set up team strategies (communication, priority of one player over others, etc.) in order to limit interference, and so, ineffective receptions. However, in such a time-constraint situation, ability to perceive and extract available information is essential, in order to better regulate self-action according to ongoing situation. Regarding this aspect, team coordination cannot be reduced to collective team-member state (DeChurch and Mesmer-Magnus, 2010), and so, to a simple sum of members' abilities.

\section{LIMITS AND PERSPECTIVES}

Although this study was one of the first to investigate the use of virtual reality to analyze coordination and cooperation in a ball interception task, it has some limitations. First, in our AL condition, balls that ended far from participants were not impossible but difficult to physically intercept. We made this choice in order to build an effective setup in SP, PP, and AP conditions: balls difficult to intercept were then intercepted by stationary (SP) or real (PP and $\mathrm{AP}$ ) partner. This resulted in trials in AL where participants did not initiate a movement of interception when the ball was very far, because they thought they could not be able to intercept it, no matter their physical involvement. As a consequence, movement initiation time may have been influenced by this absence of movement. Additionally, analysis for AL condition was made with the use of gates, and the randomness of departure and arrival positions inside the gate could have an influence on the results. We chose to base our setup on Benerink et al. (2016)'s one. However, it could be interesting to assess the influence of number of gates -and thus the use of different randomness ranges- on the results. Second, Gibson said that "the laboratory must be like life" (p. 2) (Gibson, 1979) and one could argue that our setup was not exactly a situation one could encounter in real life. When we designed the study, we tried to preserve the perception-action loop (Craig, 2013; Farrow, 2013) by involving participants in a whole-body interception. Then, although our situation was not perfectly like life, its simple design enabled us to study collaborative interaction and cooperation between two participants involved in a whole-body interception of balls. We are however aware that our experiment can be improved to be even more ecological, but we expected this study to be the first step of a new approach in the field of interaction and collaboration in virtual reality when intercepting moving objects like balls. Third, a simple avatar was used in our study. Although this choice was made to study only global interaction and limit stimulus information of our situation, it may also be interesting to explore a condition using realistic human avatar that could provide a better representation of the virtual teammate. A more realistic representation of participant's avatar could also help to assess the influence of embodiment in the virtual world (Kilteni et al., 2012). Further studies may then explore the influence of different ball speeds (and thus different time constraints), different levels of graphical realism of the avatar, other axis/plane of interception (for two participants side-by-side, intercepting balls on the mediolateral axis), or even the pattern of cooperation that emerges when more than two participants are involved in the task.

\section{CONCLUSION}

In this study, we examined the way two persons cooperate and how interference between them may occur when they perform a ball interception task, only based on visual cues. Our results showed that, when immersed in a full-body interception task (with $3 \mathrm{D}$ and stereo vision), participants self-organized their action to decide who must intercept the ball, emerging from between-participants interactions. They indeed tacitly 
divided the task so they each covered approximately $50 \%$ of space. However, interference appeared during collaboration, and the area of uncertainty observed increased when complexity of the situation increased (partner who moves and/or less visual information are available). Moreover, when assisted by a real partner, participants waited longer before initiating a movement and were influenced by the departure position of the ball: a ball starting above them was often interpreted as a ball they should intercept. Our study thus allowed to highlight this area of uncertainty and to determine factors that influence its size, i.e., its importance. Even if it uses a simple approach of collaborative-interception in VR, the design of our study allowed participants to be physically involved in the task and could thus be used for situations closer to real ones. This may be the case, for example, in various team sports where interference often occurs. To this end, we expect our work to be a new approach of interaction/cooperation in VR, and further projects may look at the influence of various parameters (such as time constraint, plane of interception, or number of participants) on the interference between participants.

\section{DATA AVAILABILITY STATEMENT}

All datasets generated for this study are included in the article/Supplementary Material.

\section{REFERENCES}

Albert, A., and Anderson, J. A. (1984). On the existence of maximum likelihood estimates in logistic regression models. Biometrika 71, 1-10. doi: 10.1093/biomet/71.1.1

Benerink, N. H., Zaal, F. T., Casanova, R., Bonnardel, N., and Bootsma, R. J. (2016). Playing 'pong' together: emergent coordination in a doubles interception task. Front. Psychol. 7:1910. doi: 10.3389/fpsyg.2016.01910

Bewick, V., Cheek, L., and Ball, J. (2005). Statistics review 14: logistic regression. Crit. Care (London, England) 9, 112-118. doi: 10.1186/cc3045

Bideau, B., Kulpa, R., Vignais, N., Brault, S., Multon, F., and Craig, C. (2010). Using virtual reality to analyze sports performance. IEEE Comput. Graph. Appl. 30, 14-21. doi: 10.1109/MCG.2009.134

Bootsma, R. J., Ledouit, S., Casanova, R., and Zaal, F. T. (2016). Fractional-order information in the visual control of lateral locomotor interception. J. Exp. Psychol. Hum. Percept. Perform. 42, 517-529. doi: 10.1037/xhp0000162

Brault, S., Bideau, B., Kulpa, R., and Craig, C. M. (2012). Detecting deception in movement: the case of the side-step in rugby. PLoS ONE 7:e37494. doi: 10.1371/journal.pone.0037494

Cañal-Bruland, R., van der Kamp, J., and van Kesteren, J. (2010). An examination of motor and perceptual contributions to the recognition of deception from others' actions. Hum. Movem. Sci. 29, 94-102. doi: 10.1016/j.humov.2009.10.001

Chardenon, A., Montagne, G., Laurent, M., and Bootsma, R. (2005). A robust solution for dealing with environmental changes in intercepting moving balls. J. Mot. Behav. 37, 52-62. doi: 10.3200/JMBR.37.1.52-62

Cooke, N. J., Gorman, J. C., Myers, C. W., and Duran, J. L. (2013). Interactive team cognition. Cogn. Sci. 37, 255-285. doi: 10.1111/cogs.12009

Cox, D., and Snell, E. (1989). The Analysis of Binary Data, 2nd Edn., Vol. 46. London: CRC Press; Chapman \& Hall.

Craig, C. (2013). Understanding perception and action in sport: how can virtual reality technology help? Sports Technol. 6, 161-169. doi: $10.1080 / 19346182.2013 .855224$

\section{ETHICS STATEMENT}

This study was carried out in accordance with the recommendations of guidelines from the Charte nationale de déontologie des métiers de la recherche of Rennes University with written informed consent from all subjects. All subjects gave written informed consent in accordance with the Declaration of Helsinki. The protocol was approved by a Rennes 2 University's local Ethics Committee.

\section{AUTHOR CONTRIBUTIONS}

$\mathrm{CF}, \mathrm{AL}, \mathrm{BB}$, and RK conceived and designed the experiments. $\mathrm{CF}$ and $\mathrm{AL}$ explored literature and analyzed the data. CF, AS, and RK conceived the virtual environment and experimental set-up. CF, AL, and TP ran the experiments. CF and RK wrote the original draft. $\mathrm{CF}, \mathrm{BB}, \mathrm{TP}, \mathrm{AS}$, and $\mathrm{RK}$ review and edited the final manuscript. All authors approved the final version of this manuscript.

\section{SUPPLEMENTARY MATERIAL}

The Supplementary Material for this article can be found online at: https:/www.frontiersin.org/articles/10.3389/fict.2019. 00020/full\#supplementary-material

Supplemental Video 1 | Video of the protocol and conditions used in the study.

Craig, C. M., Bastin, J., and Montagne, G. (2011). How information guides movement: intercepting curved free kicks in soccer. Hum. Movem. Sci. 30, 931-941. doi: 10.1016/j.humov.2010.08.007

Craig, C. M., Berton, E., Rao, G., Fernandez, L., and Bootsma, R. J. (2006). Judging where a ball will go: the case of curved free kicks in football. Naturwissenschaften 93, 97-101. doi: 10.1007/s00114-005-0071-0

Craig, C. M., Goulon, C., Berton, E., Rao, G., Fernandez, L., and Bootsma, R. J. (2009). Optic variables used to judge future ball arrival position in expert and novice soccer players. Attent. Percept. Psychophys. 71, 515-522. doi: 10.3758/APP.71.3.515

Darbutas, T., Juodžbalienè, V., Skurvydas, A., and Krišiūnas, A. (2013). Dependence of reaction time and movement speed on task complexity and age. Medicina (Kaunas, Lithuania) 49, 18-22. doi: 10.3390/medicina49010004

DeChurch, L., and Mesmer-Magnus, J. (2010). The cognitive underpinnings of effective teamwork: a meta-analysis. J. Appl. Psychol. 95, 32-53. doi: $10.1037 / \mathrm{a} 0017328$

Dessing, J. C., and Craig, C. M. (2010). Bending it like beckham: how to visually fool the goalkeeper. PLoS ONE 5:e13161. doi: 10.1371/journal.pone.0013161

Eils, E., Cañal-Bruland, R., Sieverding, L., de Lussanet, M. H., and Zentgraf, K. (2017). Vision adds to haptics when dyads perform a whole-body joint balance task. Exp. Brain Res. 235, 2089-2102. doi: 10.1007/s00221-017-4952-1

Fajen, B. R., and Warren, W. H. (2007). Behavioral dynamics of intercepting a moving target. Exp. Brain Res. 180, 303-319. doi: 10.1007/s00221-007-0859-6

Farrow, D. (2013). Practice-enhancing technology: a review of perceptual training applications in sport. Sports Technol. 6, 170-176. doi: 10.1080/19346182.2013.875031

Faure, C., Kulpa, R., Limballe, A., and Bideau, B. (2017). "Etude de la cinétique de déplacement lors d'une tâche d'interception en environnement virtuel immersif," in IGRV 2017 (Rennes).

Gibson, J. (1979). The Ecological Approach to Perception. Boston, MA: Haughton Mifflin.

Gorman, J. C. (2014). Team coordination and dynamics: two central issues. Curr. Direct. Psychol. Sci. 23, 355-360. doi: 10.1177/0963721414545215 
Hosmer, D., Lemeshow, S., Shewhart, W., and Wilks, S. (2000). Applied Logistic Regression, 2nd Edn. Hoboken, NJ: John Wiley \& Sons, Inc.

Kilteni, K., Groten, R., and Slater, M. (2012). The sense of embodiment in virtual reality. Presence 21,373-387. doi: 10.1162/PRES_a_00124

Laszlo, J. I., and Livesey, J. P. (1977). Task complexity, accuracy, and reaction time. J. Mot. Behav. 9, 171-177. doi: 10.1080/00222895.1977.10735107

Ledouit, S., Casanova, R., Zaal, F. T., and Bootsma, R. J. (2013). Prospective control in catching: the persistent angle-of-approach effect in lateral interception. PLoS ONE 8:e80827. doi: 10.1371/journal.pone.0080827

Lenoir, M., Vansteenkiste, J., Vermeulen, J., and de Clercq, D. (2005). Effects of contrasting colour patterns of the ball in the volleyball reception. J. Sports Sci. 23, 871-879. doi: 10.1080/02640410400022722

Lintern, G., Roscoe, S. N., and Sivier, J. E. (1990). Display principles, control dynamics, and environmental factors in pilot training and transfer. Hum. Fact. 32, 299-317. doi: 10.1177/001872089003200304

Meehan, M., Razzaque, S., Whitton, M. C., and Brooks, F. P. (2003). "Effect of latency on presence in stressful virtual environments," in Proceedings of the IEEE Virtual Reality, 2003 (Los Angeles, CA: IEEE), 141-148.

Niehorster, D. C., Li, L., and Lappe, M. (2017). The accuracy and precision of position and orientation tracking in the htc vive virtual reality system for scientific research. i-Perception 8:2041669517708205. doi: $10.1177 / 2041669517708205$

Orban de Xivry, J.-J., Legrain, V., and Lefévre, P. (2017). Overlap of movement planning and movement execution reduces reaction time. J. Neurophysiol. 117, 117-122. doi: 10.1152/jn.00728.2016

R Core-Team (2016). R: A Language and Environment for Statistical Computing. Vienna: R Foundation for Statistical Computing (2013).

Richardson, M. J., Harrison, S. J., Kallen, R. W., Walton, A., Eiler, B. A., Saltzman, E., et al. (2015). Self-organized complementary joint action: behavioral dynamics of an interpersonal collision-avoidance task. J. Exp. Psychol. Hum. Percept. Perform. 41:665. doi: 10.1037/xhp0000041

Rigoli, L., Romero, V., Shockley, K. D., Funke, G. J., Strang, A. J., and Richardson, M. J. (2015). "Effects of complementary control on the coordination dynamics of joint-action," in CogSci (Pasadena, CA).

Roth, D., Lugrin, J.-L., Galakhov, D., Hofmann, A., Bente, G., Latoschik, M. E., et al. (2016). "Avatar realism and social interaction quality in virtual reality," in Virtual Reality (VR), 2016 IEEE (Greenville, SC: IEEE).

Silva, P., Garganta, J., Araújo, D., Davids, K., and Aguiar, P. (2013). Shared knowledge or shared affordances? Insights from an ecological dynamics approach to team coordination in sports. Sports Med. 43, 765-772. doi: 10.1007/s40279-013-0070-9
Sofianidis, G., Hatzitaki, V., Grouios, G., Johannsen, L., and Wing, A. (2012). Somatosensory driven interpersonal synchrony during rhythmic sway. Hum. Movem. Sci. 31, 553-566. doi: 10.1016/j.humov.2011. 07.007

Varlet, M., Filippeschi, A., Ben-sadoun, G., Ratto, M., Marin, L., Ruffaldi, E., et al. (2013). Virtual reality as a tool to learn interpersonal coordination: example of team rowing. Presence 22, 202-215. doi: 10.1162/PRES_a_00151

Vesper, C., and Richardson, M. J. (2014). Strategic communication and behavioral coupling in asymmetric joint action. Exp. Brain Res. 232, 2945-2956. doi: 10.1007/s00221-014-3982-1

Vignais, N., Bideau, B., Craig, C., Brault, S., Multon, F., Delamarche, P., et al. (2009). Does the level of graphical detail of a virtual handball thrower influence a goalkeeper's motor response? J. Sports Sci. Med. 8, 501-508.

Vignais, N., Kulpa, R., Brault, S., Presse, D., and Bideau, B. (2015). Which technology to investigate visual perception in sport: video vs. virtual reality. Hum. Movem. Sci. 39, 12-26. doi: 10.1016/j.humov.2014.10.006

Vignais, N., Kulpa, R., Craig, C., Brault, S., Multon, F., and Bideau, B. (2010). Influence of the graphical levels of detail of a virtual thrower on the perception of the movement. Presence 19, 243-252. doi: 10.1162/pres. 19.3.243

Warren, W., and Fajen, B. (2008). "Behavioral dynamics of visually guided locomotion," in Coordination: Neural, Behavioral and Social Dynamics (Heidelberg: Springer), 45-75.

Watson, G., Brault, S., Kulpa, R., Bideau, B., Butterfield, J., and Craig, C. (2011). Judging the passability of dynamic gaps in a virtual rugby environment. Hum. Movem. Sci. 30, 942-956. doi: 10.1016/j.humov.2010.08.004

Wing, A. M., Endo, S., Bradbury, A., and Vorberg, D. (2014). Optimal feedback correction in string quartet synchronization. J. R. Soc. Interface 11:20131125. doi: 10.1098/rsif.2013.1125

Conflict of Interest: The authors declare that the research was conducted in the absence of any commercial or financial relationships that could be construed as a potential conflict of interest.

Copyright (c) 2019 Faure, Limballe, Sorel, Perrin, Bideau and Kulpa. This is an open-access article distributed under the terms of the Creative Commons Attribution License (CC BY). The use, distribution or reproduction in other forums is permitted, provided the original author(s) and the copyright owner(s) are credited and that the original publication in this journal is cited, in accordance with accepted academic practice. No use, distribution or reproduction is permitted which does not comply with these terms. 